\title{
An Improved Automatic Voltage Regulator for Self- Excited, Small-to-Medium Power Generating Sets equipped with Brushless Excitation Systems
}

\author{
Stefano Nuzzo \\ Department of Engineering Enzo Ferrari \\ University of Modena and Reggio Emilia \\ Modena, Italy \\ stefano.nuzzo@unimore.it \\ Michael Galea \\ Power Electronics, Machines and Control Group \\ University of Nottingham Ningbo \\ Ningbo, China \\ michael.galea@nottingham.ac.uk
}

\author{
Paolo Bolognesi \\ Electrical Machines, Power Electronics and Drives Group \\ University of Pisa \\ Pisa, Italy, \\ p.bolognesi@ieee.org \\ Davide Barater \\ Department of Engineering Enzo Ferrari \\ University of Modena and Reggio Emilia \\ Modena, Italy \\ davide.barater@unimore.it
}

\begin{abstract}
The classical, wound-field, synchronous generator is currently enjoying a revamped interest in its design and development, partly due to the ever-increasing requirements in terms of power quality standards, efficiency and power density and partly due to advances in materials and manufacturing techniques. Also, the significant improvements in the computational resources allow the utilization of modern design techniques and tools. Apart from the design of the machine itself, another area of interest is the system-level optimization. The proposed project is aimed at renewing the power electronics and the control logics in power generating sets featuring the popular brushless configuration. An industrial small-to-medium size power generating set is taken as case study. The considered platform is first analyzed at system-level, by modelling in detail all of the components comprised in it. Then, focus is given to the automatic voltage regulator. $A$ faster, more flexible and more efficient system is proposed, based on a 4-quadrant DC-to-DC converter which permits to improve the dynamic response of the excitation system.
\end{abstract}

Keywords-automatic voltage regulator, brushless excitation system, generating set modelling, synchronous generators.

\section{INTRODUCTION}

The wound-field synchronous generator represents by far the preferred choice to satisfy the electric power demand in several applications. These include power plants [1], where large machines operating in grid parallel are employed, but also generating sets (GSs), where smaller generators are used in islanded networks [2], such as boats, trains, farms, emergency systems, etc. One of the main advantages of these classical machines is the flexible and easy control of their excitation current, which permits to confront a variety of load conditions. To do so, the excitation current is varied via a dedicated excitation system. One common way of implementing such system consists in using an auxiliary generator (namely the exciter) featuring an inverted layout, whose rotating armature voltages are rectified via a rotating diode bridge feeding the main alternator field winding [3]. For isolated operation supplying passive loads, the exciter stationary field winding is properly fed by an external control system, consisting of an automatic voltage regulator (AVR), whereas for larger machines operating in parallel the control objective is usually focused on reactive power management and eventually transient damping. This system, namely brushless excitation system [4], regulates the operation of the alternator by driving the exciter field winding voltage to adjust its current and thus indirectly the field current of the main generator. The typical layout of a low-tomedium GS equipping the popular brushless excitation system is reported in Fig. 1. In such GSs, traditionally the AVRs are equipped with a power stage consisting of a simple unidirectional silicon controlled rectifier (SCR) to supply the exciter field coils. Thus, the dynamic response of the overall system results rather slow, as it involves the cascaded dynamics of an SCR rectifier, a diode rectifier and two electrical machines. In addition, when the GS is self-excited as sketched in Fig. 1, the power to the AVR is directly provided by the generator

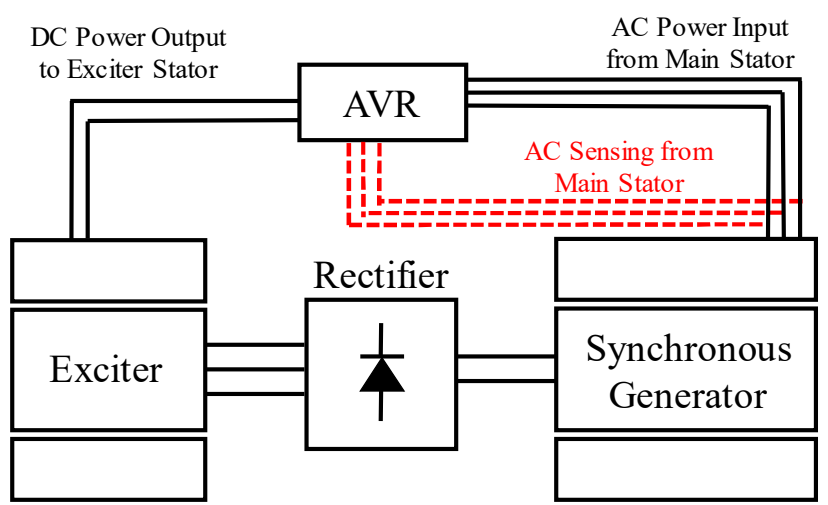

Fig. 1. Example of a brushless, self-excited GS 
terminal voltage. This means that 1) the voltage build-up for initial start-up entirely relies upon the residual magnetism of the ferromagnetic materials and 2) the AVR power and its quality strictly depend on the type of load that the generator is supplying [5]. Another challenge of this classical configuration is related to the uncontrolled nature of the commutations in the diode rectifier [6], which can produce high voltage drops (and ripples) on the DC side of the rotating rectifier (with ensuing deterioration of the GS performance). On the other hand, the brushless layout described above represents a very simple, compact and cost effective solution.

Although these systems have been extensively studied over the years, all the above implies that a significant room is still available for improving such a consolidated technology. This is also confirmed by the number of publications recently proposed in the field [7]. Besides innovative machine designs being introduced, involving both the synchronous generator and the exciter and enabled by advanced analytical, numerical and optimization techniques [8], the research community has recently focused on enhancing the dynamic performance of GSs. In particular, fully controlled rectifiers have been proposed to replace the uncontrolled rotating diode bridge currently equipping classical GSs, thus allowing a direct control of the generator field winding and in turn enabling the development of permanent magnet (PM) exciters [9]. While these solutions result in much more performing systems, however they come at the cost of increasing the overall complexity and cost. A hybrid PM - field winding exciter has been proposed in [10], which achieves an improved overall system efficiency while limiting the production costs. A significant constraint of this work was that of keeping the rest of the system unaltered, i.e. the standard diode rectifier and SCR-based AVR were used. However, due to inherent 2-quadrant operation of the SCR, only positive current values can be provided to the exciter field winding. Therefore, the magnets were designed to provide the rated voltage at the generator terminals when this operates at no-load. On the other hand, the constraint imposed in [10] limits the degrees of freedom relative to the exciter design, which can be further improved by selecting a more suitable operating point for the sizing of the PMs. In fact, when any of the generator load condition is assumed for the PM design, the unidirectional SCR is no longer sufficient and a static converter able to provide also negative current values to the exciter field coils needs to be used.

In this context, this paper proposes an improved AVR power stage that, besides potentially allowing to increase the design degrees of freedom mentioned above, can enhance the dynamic performance of classical GSs equipped with standard woundfield exciters. An off-the-shelf 400-kVA platform is considered as case study. First, its SCR-based AVR behaviour is investigated at system-level, i.e. its model is studied in terms of interaction with the exciter, the diode rectifier and the main generator models. After highlighting the limitations of the benchmark system, a number of solutions are investigated. Finally, the most flexible configuration in terms of voltage and current requirements is chosen to replace the SCR-based AVR, consisting of a 4-quadrant DC/DC converter. The selected topology achieves improved performance compared to the benchmark system and, to the authors' perspective, it suits perfectly the new requirements and design alterations that the classical GSs are currently enjoying.

\section{System Modelling Methodology}

Before proposing possible improvement solutions, a comprehensive and accurate system-level modeling methodology for classical GS that include machines, converters and control considerations is required. Therefore, in this section, the models of each component comprised within the GS under study are described. The modeling environment chosen for the analysis is Matlab-Simulink.

\section{A. The exciter and the main synchronous generator}

Usually, in system-level models of power GSs, simplified d$\mathrm{q}$ approaches based on sinusoidal approximation of the electromagnetic quantities are adopted for the machines involved, i.e. generator and exciter. However, this assumption is often far from the real behavior of these electrical machines. This is also the case for the platforms investigated in this work. In fact, the geometrical and winding structures featured by both the exciter and the synchronous generator do not allow to assume sinusoidal shape of the relevant quantities. This is especially true for the exciter machine, where the 14 stator poles feature a non-optimised shape and the armature winding has a single-layer layout and is characterised by 1 slot-per-pole-perphase only, making the output voltage waveforms significantly rich of harmonics. On the other hand, the main generator is a 4pole machine, equipped with 4 rotor salient poles with 6 damping bars-per-pole and a double-layer armature winding with 4 slots-per-pole-per-phase and a short pitching equal to $1 / 3$ of the pole pitch. In this work, all of these geometrical and winding characteristics are taken into account in detail by means of step-like permeance and winding functions, for both the machines comprised in the GS. The detailed description of the exciter and of the synchronous generators at hand can be found in [2] and [3], respectively. The details, the assumptions and the full description of the analytical model used for analyzing the aforementioned machines can be found in [11]. In [12], the method is applied to the synchronous generator being studied in this paper. An innovative damper cage modelling approach is proposed in [12], allowing to significantly reduce the system unknowns and thus its complexity. The overall analytical methodology is based upon the calculation of the inductances of the machine as only dependent on the position of rotor vs. stator, i.e. the linear behaviour of the ferromagnetic materials is assumed. The same approach is adopted for the exciter and the main generator in this work.

The state variables employed for the electromagnetic analysis are the currents flowing through the machines' phases. The vector containing these currents is indicated as $\bar{l}$ in Fig. 2, where the general scheme of the machines' models implemented in Simulink is shown. The number of state variables is 4 for the exciter (i.e. 1 for its field winding and 3 for its armature) and 9 for the main alternator (i.e. 1 for its field winding, 3 for its armature and 5 for its damper cage). The information relative to the resistive and inductive circuital aspects of both the machines ( $R$ and $L(\alpha)$ in Fig. 2) are first calculated off-line in Matlab and then stored in look-up tables in Simulink for the numerical resolution. The additional branch $Z_{\text {add }}$ is only used in the generator model and it represents the load supplied by the 
machine itself, whereas the exciter armature phases are connected to the diode rectifier, whose model will be described in the next sub-section.

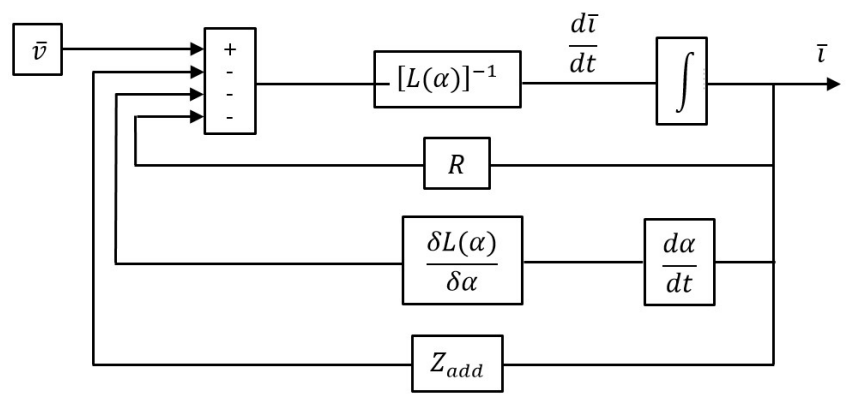

Fig. 2. General scheme of the circuital aspects relative to the exciter and the main generator.

\section{B. The diode rectifier}

The six-pulse diode rectifier has to manage the 3 state variables deriving from the exciter armature. To do so and to allow for the separation between state variables, the upper diodes (indicated as $\mathrm{D}_{\text {up }}$ in Fig. 3) of each leg are modeled through saturation and gain blocks to reproduce their classical voltage/current characteristics, whereas the lower diodes (indicated as $\mathrm{D}_{\text {lw }}$ ) of each leg are modeled by adding a capacitance $\mathrm{C}_{\mathrm{p}}=1 \mu \mathrm{F}$ in parallel, so that the state variables become the voltages $\mathrm{V}_{\mathrm{Cp}}$ across them. The circuital component $\mathrm{C}_{\mathrm{p}}$ can be interpreted as the parasitic capacitance of the diodes. For the upper diodes, taking into account that the rated full-load field current of the main generator is $50 \mathrm{~A}$ and assuming a forward voltage of $0.7 V$ for the diodes, the gain $\mathrm{k}_{\mathrm{p}}=50 / 0.7$ is used for the first quadrant portion of the voltage/current characteristic, whereas a gain value $\mathrm{k}_{\mathrm{n}}=\mathrm{k}_{\mathrm{n}} / 10000$ is chosen for the third quadrant portion.

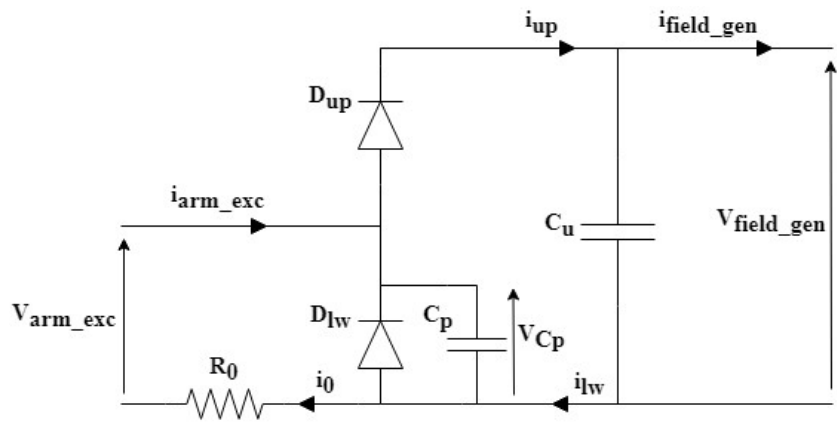

Fig. 3. Single-phase schematic of the diode rectifier model.

Considering that the rectified current feeds the synchronous generator field winding and that such current is one of the state variables of the alternator model (see Section II.A), a capacitance $\mathrm{C}_{\mathrm{u}}=4.7 \mathrm{mF}$ is added in parallel to the load supplied by the diode bridge to allow for a further separation of the state variables. This modelling choice however transforms the generator field winding in a voltage-stiff system, while by nature it is often a current-stiff one. Hence, such large capacitance can affect the dynamic response of the GS. On the other hand, it is worth highlighting that this works aims at comparing the original AVR with the one proposed and described in Section III. This means that, as far as the same modelling choice is adopted also for the model of the GS comprising the proposed AVR solution, the comparative analysis should not be compromised. In addition, the inclusion of a suitable capacitance between the diode rectifier and the generator field winding should be considered as a possible design choice, when the voltage ripple reduction becomes a main objective.

All of the circuital aspects discussed above are illustrated in the single-phase (i.e. referred to one single leg of the diode bridge) scheme of Fig. 3, where a high value neutral resistance, i.e. $\mathrm{R}_{0}=0.1 M \Omega$, can be also observed. This neutral resistance was added to ensure a balanced distribution of the exciter armature currents.

\section{The SCR-based AVR}

The SCR-based AVR consists of a thyristor that delays the firing to control the exciter field voltage. A freewheeling diode in anti-parallel with the SCR output ensures a freewheeling path for the field current while the SCR device is blocked. The input parameter of this circuital layout is the line-to-line voltage $\mathrm{V}_{\text {arm gen }}$ of the main generator. The thyristor, indicated as SCR in Fig. 4, is modeled by using the current isCR across it as state variable. To do so, a small inductance $\mathrm{L}_{\mathrm{SCR}}=10 \mu \mathrm{H}$ is added in series to the thyristor. $\mathrm{L}_{\mathrm{SCR}}$ can be interpreted as the parasitic inductance of the device. The freewheeling diode $\mathrm{D}_{\mathrm{fw}}$ is modeled with a small capacitance $\mathrm{C}_{\mathrm{fw}}=1 \mu F$ in parallel, as seen in Fig. 4. This can be interpreted as the parasitic capacitance of the diode. Therefore, $\mathrm{C}_{\mathrm{fw}}$ implies that the assumed state variable is the voltage $V_{\text {field_exc }}$ across it, thus permitting its separation from the state variables employed in the exciter model, i.e. the currents flowing in the machine phases, obviously including the field winding. The voltage $\mathrm{V}_{\text {field_exc }}$ is thus used as input for the exciter model. The circuital aspects discussed above are summarised in Fig. 4. As additional input for the AVR model, a gate voltage signal is implemented to control the firing angle. This gate voltage is controlled by a PI controller according to the procedure discussed in the next sub-section.

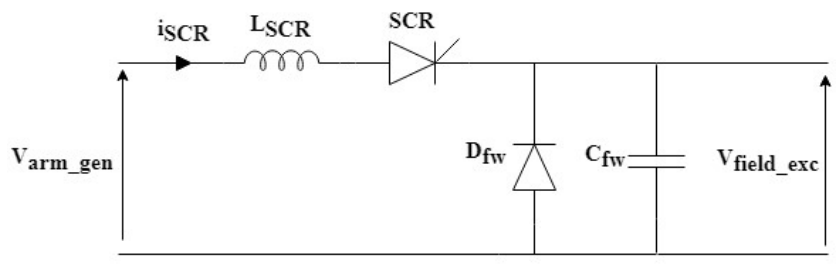

Fig. 4. Simplified schematic of the benchmark SCR-based AVR.

\section{The control objective and the whole GS model}

In a self-excited GS, the control objective is that of maintaining the generator terminal voltage at a pre-set constant value. Therefore, in the system under investigation, the output voltages of the synchronous generator model are registered and compared against a reference value. To do so, first low pass filters with a frequency bandwidth of $70 \mathrm{~Hz}$ are used to obtain only the fundamental harmonics (i.e. at $50 \mathrm{~Hz}$ ) of any of the 3 line-to-line voltages. Then, the relevant RMS values are determined and averaged to be compared vs. the reference signal. The error between the signals is elaborated by a standard 
PI controller, whose output is opportunely manipulated to adjust the gate voltage of the SCR-based AVR model described above. The entire GS block diagram comprising all of the subparts described in the previous sub-sections is shown in Fig. 5.

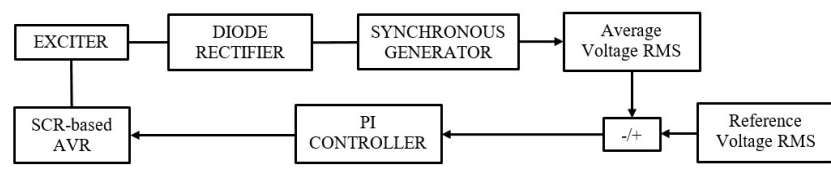

Fig. 5. Block diagram of the GS model built in Simulink.

\section{PossiBle ImPROVEMENT OPtiONS AND FinAL SOLUTION}

\section{A. General Considerations}

One of the main drawbacks of the classical brushless excitation system investigated in this work is the indirect control of the generator field current. This is due to the inherently uncontrolled nature of the rotating diode bridge. Therefore, a number of configurations which allow to "directly" control the alternator field current have been recently proposed, thus potentially permitting to achieve better waveforms for improved steady-state power quality and faster dynamic responses to manage more effectively any transient condition. These solutions entail the replacement of the passive diode rectifier with some sort of active converter. The simplest layout implements a thyristor bridge rectifier. More sophisticated and faster systems can include 1) a diode rectifier supported by a dual-quadrant DC-to-DC converter, 2) a rotating Vienna rectifier with a dual-quadrant DC-to-DC bridge or 3) a rotating inverter [9]. All of the described options permit to use PM exciters and the voltage regulation task is accomplished by wireless signal transmission between stator and rotor. These solutions present several benefits compared to the classical layout, including lower ripple of the rectified field voltage, faster dynamic response, enhanced controllability, improved reliability and overall performance. However, due to the elevated costs of the topologies proposed as alternatives to the popular system implementing wound-field exciters and diode rectifiers, GS manufactures are still reluctant to such improvement changes.

On the other hand, little focus has been given to the AVR power stage. It is perceived that, at least for the management of the slow dynamics, the AVR layout can play an important role within the GS. Also, any major modification to the AVR will not be significantly disruptive from a cost perspective, as the main core of the whole system remains unaltered. In fact, the
AVR circuitry is stationary (as opposed to the converter interposed between exciter and alternator) and no wireless technologies are necessary for signal transmission.

The first limit to highlight for the benchmark SCR-based AVR is its unidirectional operation, meaning that it is able to provide only positive values of the exciter field current. Therefore, besides possibly improving the GS dynamic performance, a more flexible operation would enable further system improvements. In fact, when a hybrid PMs - field winding solution is envisioned for the exciter as proposed in [10], the unidirectional nature of the SCR limits the degrees of freedom of the PMs design. In other words, they have to be sized in such a way to provide an excitation level (when no current flows in the exciter field winding) which would not imply the need for negative exciter field currents at any of the operations of the main generator. For example, in [10], the rated no-load operation of the alternator was targeted for the PMs design. On the other hand, the implementation of a bi-directional system feeding the exciter field winding would permit to size the PMs in such a way to provide an excitation level (when no current flows in the exciter field winding) targeting any of the load operations points of the main generator. In fact, in such a scenario, even in the case of a de-excitation requirement a negative current could be always provided to the exciter, just thanks to the use of a bi-directional converter..

\section{B. The proposed AVR}

Given the above observations, a 4-quadrant DC-to-DC converter is selected as replacement for the SCR. Considering that the AVR power is provided by the generator line-to-line terminal voltage $\mathrm{V}_{\text {arm gen }}$, an additional circuitry, consisting of a power factor correction (PFC) stage, is introduced to opportunely create the input DC link for the DC-to-DC converter. The PFC circuitry was selected for its relatively simple layout and to limit the currents' harmonic content of the on the AC side. The proposed topology is seen in Fig. 6.

The Graetz bridge (including the diodes $\mathrm{D}_{1}, \mathrm{D}_{2}, \mathrm{D}_{3}$ and $\mathrm{D}_{4}$ ) is modeled considering that 1 ) its output voltage is equal to the absolute value of its input voltage, 2) its output current is either equal or opposite to its input current when the input voltage is positive or negative, respectively. In order to generate the DC link voltage $\mathrm{V}_{\mathrm{PFC}}$ shown in Fig. 6, a hysteresis controller is implemented to control the group " $L_{P F C}-T_{P F C}-D_{P F C}$ " preceding it. The state variables employed here are the current $i_{\mathrm{PFC}}$ across the inductance and $\mathrm{V}_{\mathrm{PFC}}$. The latter is set in first approximation at $100 \mathrm{~V}$ and is obtained through a standard PI controller which elaborates the error between the DC link voltage and a reference

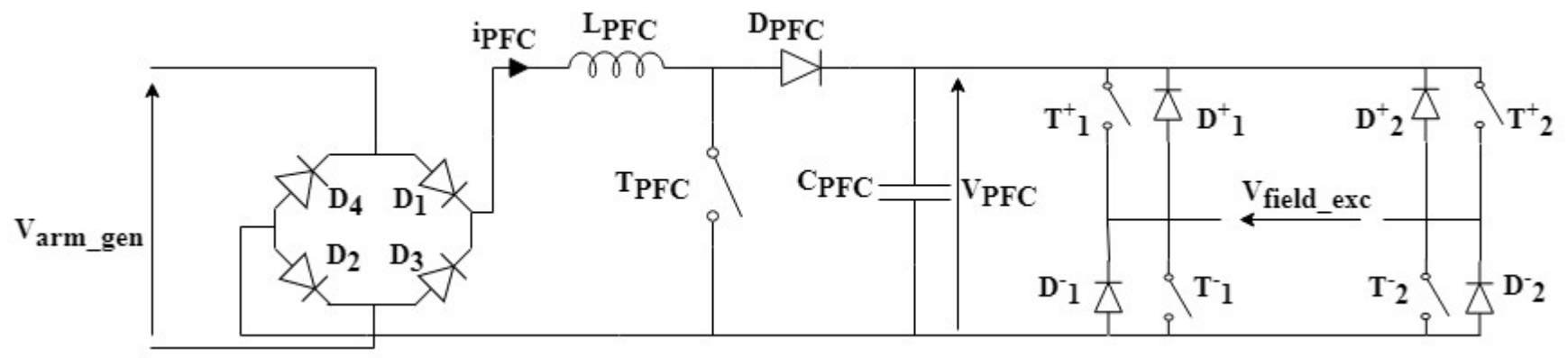

Fig. 6. DC link derived from the rectified input voltage and selected 4-quadrant DC-to-DC converter feeding the exciter field winding. 
value. The output voltage and current of the DC link model represent the input parameters for the 4-quadrant converter. Its 2 legs are controlled separately by simple relationships between input and output quantities. The control input for both legs is given by a reference current profile opportunely generated by the main PI controller which elaborates the error between reference and measured RMS values of the generator line-to-line terminal voltage, which represents the control objective.

\section{DYNAMIC PERFORMANCE COMPARISON}

Having provided the circuital and modeling aspects of both benchmark and proposed AVRs for the GS under analysis, the next and conclusive step consists in proving the dynamic performance improvements achieved by the configuration of Fig. 6. The comparison in is performed at no-load operation. This is simulated by choosing a diagonal form of the matrix $Z_{\text {add }}$ introduced in Section II.A (see Fig. 2), whose elements feature very high values (set at $10 \mathrm{k} \Omega$ in the model). For both the models, the voltage RMS reference value is set at $150 \mathrm{~V}$. The dynamic responses of the SCR-based and of the DC-to-DC converterbased AVRs can be observed in Fig. 7. As perceived, the proposed system significantly improves the dynamic performance, achieving a much lower overshoot in the first instants and a perfect match between reference and measured signals at steady-state. To understand these achievements, a comparison between the exciter field currents provided by the benchmark and the proposed AVRs is reported in Fig. 8. The faster response of the proposed system allows for the reduction of the overshoot in the voltage RMS. In addition, the significant current ripple reduction observed in Fig. 8 is responsible for the absence of RMS voltage ripple in the upgraded GS (see Fig. 7).

\section{A. Discussion}

All the benefits highlighted above for the proposed AVR with respect to the original system come at the cost of increasing the losses in the switching components. In addition, the DC-toDC converter is not as cost effective as the SCR-based AVR. The added losses and costs need however to be "weighted" against the potential advantages resulting from the adoption of the proposed AVR over the entire lifetime of the GS. More investigations on these aspects will be carried out in future research.

Besides the above, more future work would include

1) The detailed analysis of the SCR based and of the DCto-DC converter based AVRs will be carried out by using specific tools such as PLECS or Simscape;

2) An optimal hybrid PM - field winding exciter will be designed and prototyped leveraging on the bi-directional capabilities of the proposed converter;

3) The DC-to-DC converter will be sized and prototyped according to the new power requirements of the optimal hybrid exciter discussed in Point 2).

4) The proposed exciter and converter will be tested on a full-scale GS to highlight all of the potential benefits discussed throughout the paper.

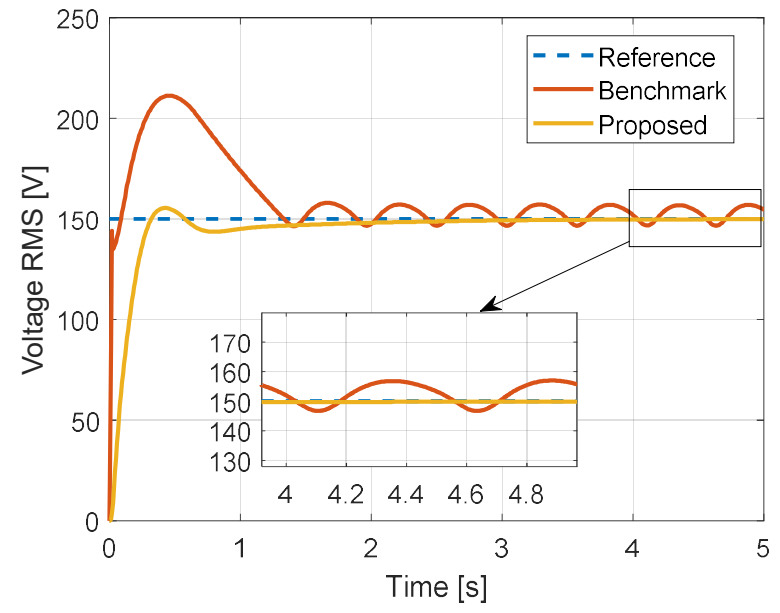

Fig. 7. Comparison between benchmark and proposed systems - voltage RMS response at no-load operation of the main generator.

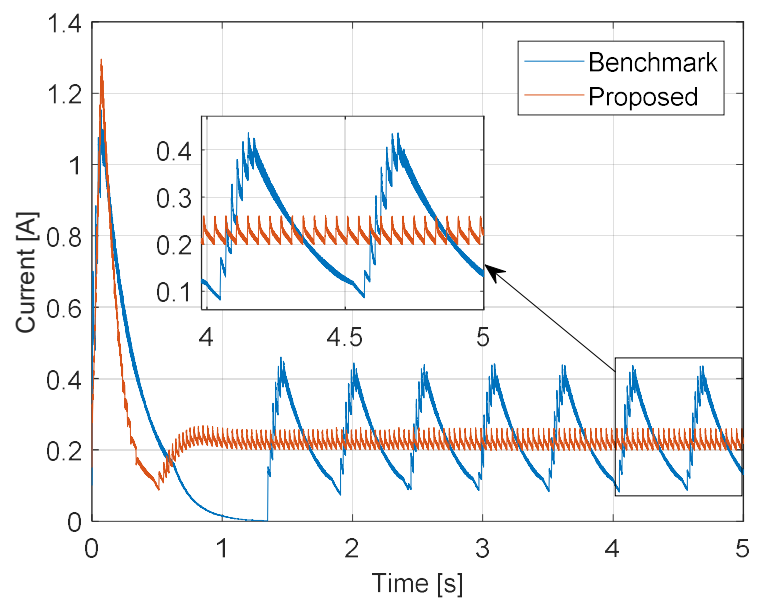

Fig. 8. Comparison between benchmark and proposed systems - exciter field current resulting from the AVR output.

\section{CONCLUSIONS}

This work has presented a possible improvement option for the automatic voltage regulator system of an off-the-shelf, 400kVA generating set, in line with the growing trend of renovation which is positively affecting these classical systems. The benchmark generating set was modeled in the MatlabSimulink environment through a comprehensive and multidisciplinary approach. This is able to simulate in detail the electrical machines, the power electronics and the control strategies implemented within the whole system. A number of possible improvement options were discussed, highlighting positive and negative implications. Focus was then given to the AVR power stage, given its reduced impact on the overall cost and complexity of the system.

The benchmark SCR-based AVR was superseded by a more flexible 4-quadrant DC-to-DC converter, whose input DC link was opportunely identified and studied. Finally, a performance 
comparison in terms of step dynamic response was carried out, showing very promising results of the proposed topology.

To the authors' perspective, the proposed AVR layout suits perfectly the recent advancements in the field of small-tomedium power generating sets and can represent an important candidate for the generating set of the future, which involves hybrid-excited designs, wireless technologies, etc.

\section{ACKNOWLEDGMENT}

This work was funded by the EPSRC Centre for Power Electronics through research grant [EP/K035304/1].

\section{REFERENCES}

[1] J. Han, W. Li, Y. Li, "Analysis of Three-Dimensional Complex Fluid Flow and Temperature Distribution in the End Region of a Turbogenerator," IEEE Transactions on Industrial. Electronics, vol. 62, no. 9 , pp. 5370-5381, 2015.

[2] S. Nuzzo, P. Bolognesi, G. Vakil, D. Fallows, C. Gerada, N. L. Brown, M. Galea, "A methodology to remove Stator Skew in Small-Medium Size Synchronous Generators via innovative damper cage designs," DOI 10.1109/TIE.2018.2864699, IEEE Transactions on Industrial Electronics, 2018.

[3] S. Nuzzo, M. Galea, C. Gerada and N. Brown, "Analysis, Modelling and Design Considerations for the Excitation Systems of Synchronous Generators", IEEE Transactions on Industrial Electronics, vol. 65, no. 4, pp. 2996-3007, 2018.

[4] T. L. Dillman, F. W. Keay, C. Raczkowsi, J. W. Skooglund and W. H. South, "Brushless excitation” IEEE Spectr., vol. 9, no. 3, pp. 58-66, Mar. 1972.
[5] G. Laliberte, "A comparison of generator excitation systems (Power topic 6008 - Technical information from Cummins Power Generation)," in Technical Report GLPT-6008-EN (10/14), Oct 2014, pp. 1-6.

[6] S. Nuzzo, M. Galea, C. Gerada and N. L. Brown, "Prediction of the voltage drop due to the diode commutation process in the excitation system of salient-pole synchronous generators," 2016 19th International Conference on Electrical Machines and Systems (ICEMS), Chiba, 2016, pp. 1-6.

[7] J. K. Nøland, F. Evestedt, J. J. Perez-Loya, J. Abrahamsson, and U. Lundin, "Design and characterization of a rotating brushless outer pole PM exciter for a synchronous generator," IEEE Trans. Ind. Appl., vol. 53 , no. 3, pp. 2016-2017, May./Jun. 2017.

[8] G. Bramerdorfer, J. A. Tapia, J. J. Pyrhönen and A. Cavagnino, "Modern Electrical Machine Design Optimization: Techniques, Trends, and Best Practices," in IEEE Transactions on Industrial Electronics, vol. 65, no. 10, pp. 7672-7684, Oct. 2018.

[9] J. K. Nøland, S. Nuzzo, A. Tessarolo, E. F. Alves, "Excitation System Technologies for Wound-Field Synchronous Machines: Survey of Solutions and Evolving Trends," IEEE Access, vol. PP, pp. 1-19, 2019.

[10] G. Decuzzi, S. Nuzzo, P. Bolognesi, P. Giangrande, M. Galea, "Hybrid Magnet - Field Winding Solutions for Exciters of Synchronous Generators", in Proceedings of 2019 IEEE International Electric Machines and Drives Conference (IEMDC), pp. 1458-1463, 2019.

[11] P. Bolognesi, "A mid-complexity analysis of long-drum-type electric machines suitable for circuital modelling," Proc. of ICEM 2008 Conf., Vilamoura, Sep. 2008.

S. Nuzzo, P. Bolognesi, M. Galea, C. Gerada, "Simplified Damper Cage Circuital Model and Fast Analytical-Numerical approach for the analysis of Synchronous Generators", IEEE Transactions on Industrial Electronics, pre-print DOI 10.1109/TIE.2018.2885737 